\title{
SUMMER MEETING AT CHICAGO CONFERENCE REPORTS
}

\author{
CONFERENCE ON ALGEBRA AND TWENTY-THIRD COLLOQUIUM
}

The Conference on Algebra at the 1941 Summer Meeting of the Society in Chicago was held in three sessions. The first dealt with abstract algebra, especially with lattice theory, and the lectures were given by Professors John von Neumann and Garrett Birkhoff. The session was preceded by the first Colloquium Lecture of Professor Oystein Ore on the allied topic of Mathematical Relations and Structures. The second session was concerned with topics in linear algebra and the theory of matrices and the speakers were Professors Nathan Jacobson and N. H. McCoy. General arithmetic notions came to the fore in the third and final session, with talks by Professors J. F. Ritt and Oscar Zariski, and Dr. O. F. G. Schilling. There was considerable discussion, both organized and spontaneous, after all the talks.

\section{Abstract algebra and lattices}

A number of different algebraic systems can be subsumed under the notion of a lattice-ordered group (or an l-group). Professor Birkhoff's talk dealt with the structural properties of such groups. By definition, an $l$-group is a group which is also a lattice and which has the "homogeneity" property ( $x \leqq y$ implies $a+x \leqq a+y$ and $x+a \leqq y$ $+a)$. Examples include the additive groups of ordered fields, partially ordered function spaces (Kantorovitch and others), and the lattice of all ideals in an integral domain (Clifford, Lorenzen, Krull).

After discussing the elementary properties of $l$-groups and the variety of possible postulate systems, Birkhoff turned to their structure theory. The study of homomorphisms of $l$-groups leads naturally to a concept of an $l$-ideal. The lattice of all $l$-ideals is distributive. A central result asserts that every $l$-group with a chain condition is either directly decomposable, or has exactly one maximal proper $l$-ideal.

Many of the results known for abelian $l$-groups hold without serious change for non-abelian groups. However, any $l$-group in which there is a chain condition for the elements is necessarily abelian. There is a number of unsolved questions: Does there exist a non-abelian $l$-group which is complete, in the sense that every bounded set of elements has a least upper bound? Is there a theory of $l$-rings? Are there simple (non-abelian) $l$-groups which are not simply ordered?

von Neumann began by a comparison of classical and quantum 
mechanics. Any two operators (observables) of classical mechanics can be added and multiplied together. But in quantum mechanics, the observables, being hermitian operators, can be added but not multiplied together, unless they are permutable. Hence they form a commutative group only, and not a ring. This group is partially ordered if the positive semi-definite operators are called "positive." Unfortunately, this ordering does not have the lattice property, again unlike classical mechanics. By assuming the lattice property of classical mechanics, but not the ring property, we get a physical background for the study of complete lattice-ordered abelian groups which are not rings.

These have very special structure. Consider the complete modular lattice $M$ of all subgroups of such a group $G$. In $M$, the $l$-ideals of $G$ form a distributive sublattice $D$, closed in $M$ with respect to unrestricted unions and intersections. The closed $l$-ideals form a complemented sublattice $B$ of $D$ (hence a Boolean algebra), which is closed in $D$ under unrestricted intersections, but only under finite unions.

Furthermore, using $B$ and the representation theory of Boolean algebras, due to Stone, by bicompact totally disconnected topological spaces, one can obtain a representation theory for $G$ by real functions on such spaces. In this representation, addition corresponds to addition; however, a function is to be called positive if the set on which it assumes negative values is nowhere dense. At any point, a function may assume either all integral or all real values.

\section{Linear algebras and matrices}

Many of the properties of matrices ordinarily proved only for matrices with elements in a field can actually be stated and proved, in appropriate form, for matrices whose elements lie in more general rings. McCoy's conference lecture was concerned with results of this character, especially for noncommutative rings. One of the accomplishments of the E. H. Moore General Analysis was a definition of a determinant for a hermitian matrix whose elements are quaternions. The same technique can be applied to define the determinants of suitably restricted matrices over much more general noncommutative rings. However, the proofs of some of the appropriate properties of these determinants require new devices.

The determinant can be used to define a characteristic polynomial of a matrix $A$ and to prove the analogue of the Cayley-Hamilton theorem that every matrix satisfies its characteristic equation. The minimal polynomial of an ordinary matrix is replaced by a suitable minimal ideal, composed of all polynomials of which the matrix is a 
root. In terms of the elements of the adjoint matrix one can determine a condition of the usual form that a polynomial belong to this minimal ideal. The theorems of Phillips and Ostrowski about equations satisfied by several matrices can also be generalized to matrices with elements in a ring.

Jacobson's talk dealt with Lie Algebras. Let $A$ be an associative algebra of characteristic $p$. It is well known that the elements of $A$ form a Lie algebra if one defines $[a b]=a b-b a$. Jacobson pointed out that in addition, the following identities hold

$$
\begin{aligned}
& (a+b)^{p}=a^{p}+b^{p}+s(a, b), \\
& {[\cdots[[a b] b] \cdots b]=\left[a b^{p}\right],}
\end{aligned}
$$

provided $s(a, b)$ is a suitably defined, but complicated, Lie polynomial in $a$ and $b$. He defined a restricted Lie algebra of characteristic $p$ as a Lie algebra with operation $a \rightarrow a^{p}$ which satisfies (I)-(II) and $(\alpha a)^{p}$ $=\alpha^{p} a^{p}$.

He mentioned, as other examples of restricted Lie algebras, the skew-symmetric elements of $A$ under any anti-automorphism of $A$, and the "derivations" of any algebra (associative or not). A derivation is a linear operator $D$ which satisfies, for all $x, y,(x y) D=(x D) y$ $+x(y D)$, and corresponds, over the real field, to an infinitesimal automorphism.

Conversely, Jacobson showed that any restricted Lie algebra $L$ could be realized by a Lie subalgebra of a suitably constructed associative algebra $U_{L}$. (The corresponding result for ordinary Lie algebras was known.) Sharper results were given. The subalgebra consists precisely of those elements of $U_{L}$ which are skew under a certain involution of $L$. Moreover, if $L$ has a finite basis, then so does $U_{L}$; hence $L$ has only a finite number of inequivalent irreducible representations by matrices. If, in addition, every element of $L$ is nilpotent, then $U_{L}$ is a nilpotent associative algebra.

Finally, Jacobson showed that every derivation of $U_{L}$ induced a "restricted" derivation of $L$, in the sense that $x^{p} D=[\cdots[x D, x]$ $\cdots x]$, and conversely, every restricted derivation of $L$ was induced by some derivation of $U_{L}$. These results have impelled him to the conclusion that restricted Lie algebras of characteristic $p$ are closer analogues of Lie algebras of characteristic $\infty$ than ordinary (unrestricted) Lie algebras of characteristic $p$.

\section{General arithmetic}

The theory of polynomial ideals is concerned essentially with the manifold of solutions of polynomial equations in many variables, 
but there has been developed a parallel theory for formal differential equations and the corresponding manifolds of solutions. Ritt's lecture summarized some of the recent results in this theory, emphasizing the respects in which this theory diverges from the polynomial case.

Starting with a form $F$ which is a polynomial in the unknowns $y_{1}, \cdots, y_{n}$ and their derivatives, with coefficients in some (differential) field $K$, there is a corresponding manifold $M$, which consists of all the zeros $a_{1}, \cdots, a_{n}$ of $F$ lying in some extension of the coefficient field $K$. There is a unique decomposition of such a manifold into "irreducible" manifolds, which corresponds to the decomposition of a "perfect" differential ideal into an intersection of prime ideals. However, there is no exact analogue of the ordinary algebraic theorem which gives a decomposition of arbitrary ideals into primary components. The dimension of an irreducible manifold can be appropriately defined, but the dimension of an intersection does not behave properly. Among the irreducible components of the manifold of a form, one is essential, in the sense that it does not occur in the manifolds of other forms. Ritt quoted theorems giving conditions that a given manifold be essential for a form in one variable. These conditions, originally found by analytic methods, have been derived in part by purely algebraic arguments, in a recent paper by $\mathrm{H}$. Levi. The discussion after the lecture concerned some new basis theorems for systems of forms over fields of prime characteristic.

The famous and difficult problem of the reduction of the singularities of an algebraic manifold by birational transformations was the subject of Zariski's conference lecture. An $r$-dimensional manifold is given by a field of algebraic functions of $r$ independent variables. Any $n$ elements $x_{1}, \cdots, x_{n}$ which generate this field determine a projective model of the manifold in the $n$-dimensional projective space with the (nonhomogeneous) coordinates $x_{1}, \cdots, x_{n}$. The problem consists in finding some model of the manifold in which there will be no singular points.

Zariski has published an algebraic solution of this problem, for twodimensional manifolds. His talk discussed the generalization of this attack to higher dimensions. Two steps are necessary. The first is that of local uniformization of the "places" of the manifold. A place is essentially a valuation of the associated field of algebraic functions. The uniformization theorem asserts that there is a projective model of the manifold on which the "center" of the valuation is a simple point. This step has been carried out for $r$-dimensional manifolds.

The second step is that of getting this uniformization to hold simultaneously for all places. Zariski discussed the procedures available 
here, which consist essentially in a suitable application of locally quadratic transformations. He announced that his methods now were sufficiently powerful to solve the problem of reducing the singularities of a three-dimensional manifold, in the case where the field of coefficients is the complex number field.

Schilling's talk dealt with a generalized Hilbert theory for fields with a valuation. The original Hilbert theory concerns the successive stages in the decomposition of a rational prime $p$ into prime ideals in a normal algebraic number field. Alternatively, one may start, not with the field of rational numbers, but with the associated $p$-adic number field. In the general case the prime $p$ is replaced by a valuation, and one requires that the field $F$ be relatively complete with respect to this valuation (this assumption is more appropriate than the stronger requirement of topological completeness). Any normal extension of such a field $F$ may be obtained in two uniquely determined steps. The first step is an "inertial" extension, in which the valuation is unramified (that is, the corresponding prime ideal is not decomposed). The second step is a completely ramified extension, in which the residue class field is not extended. There is a corresponding decomposition of the Galois group into two parts. The whole theory can be carried through for infinite extensions, and the resulting Galois groups can be explicitly characterized. In case the residue class field is finite, and the order of the group is a power of a prime, the reverse problem can be solved: one obtains necessary and sufficient conditions that a given group can be realized as the Galois group of a normal extension of the given base field. The whole theory is analogous to a generalized "Kummer theory" for function fields.

\section{The Colloquium Lectures}

The object of these lectures is to make a general study of the properties of mathematical relations as they occur in the various mathematical theories. One may illustrate such relations by various examples, for instance, the relations: one element contains another, a point is middle point between two given ones, a set is the closure of another set. One finds that a more general type of relations may be considered as correspondences $A \rightarrow R_{A}$ between sets, where the set $R_{A}$ corresponding to a set $A$ may depend not only upon the elements in $A$, but also upon a certain grouping and ordering of the elements in $A$, as, for instance, in the case of noncommutative or non-associative multiplication. If an element $a$ in a set $S$ belongs to the subset $R_{A}$ it is convenient to write this as a relation $a R A$. Such relations may be combined in various ways and certain normal forms of relations may 
be introduced. Particularly interesting is the problem of finding automorphisms or endomorphisms of a relation $R$, namely those one-toone or many-to-one correspondences $\alpha$ such that any relation $a R A$ is taken into another valid relation $a^{\alpha} R A^{\alpha}$. The determination of all automorphisms is connected with the problem of finding all correspondences commuting with a given correspondence. It should also be mentioned that these problems lead to a study of the so-called monomial groups and to problems connected with a general Galois theory, extending the ordinary Galois theory of equations.

Of particular importance are the so-called binary relations $a R b$ between two elements $a$ and $b$. As examples, one may think of $a$ greater than $b, a$ equivalent to $b, a$ orthogonal to $b, a$ divides $b$, and so on. The theory of such binary relations may be conceived of in many ways. The first systematic theory of such binary relations was given by Peirce and by Schroeder. In their formulation one can say that the theory appears as a theory of matrices over a Boolean ring. Another method of representing the binary relations consists in conceiving the relation as a graph, joining two vertices $a$ and $b$ by an edge from $a$ to $b$ whenever $a R b$. The symmetric relations are those for which $a R b$ imply $b R a$. They correspond to the symmetric matrices or to graphs in which the edges are considered undirected.

When the relations are considered to be matrices one can also unite their theory with vector spaces over special rings. For the relations one can define various operations: sum and intersection, multiplication and dual multiplication. Thus it becomes possible to introduce a ring and ideal theory of binary relations and certain parts of the ordinary theory of linear algebras carry over to relations. In other ways the theory of relations differs considerably from ordinary matrix theory. Also here one is interested in the automorphisms and endomorphisms of the relations. It turns out that the automorphisms correspond to the permutation matrices which commute with the relation matrix, and this property may be used for the determination of automorphisms.

Of particular interest in the theory of relations are the transitive relations for which

$$
a R b, b R c \text { implies } a R c \text {. }
$$

These relations may be characterized in the ring of all relations in various ways, for instance, by the property $R^{2} C R$ or also as certain units or as certain sum relations. They are closely associated with the idempotents of the rings of relations. Any transitive relation can be broken up in an equivalence relation and a partial order relation. For 
the equivalence relations a number of important algebraic problems can be solved completely, and the interest is therefore centered upon the partially ordered sets. These sets have gained an increasingly important position in mathematical theories, and a number of useful properties of these sets are known. Only certain aspects of these theories are discussed in detail in these lectures.

Among these partially ordered sets the lattices or structures are particularly important. In these systems there exists a unique minimal element $a \cup b$ containing any two elements $a$ and $b$, and a unique maximal element $a \cap b$ contained in both. These elements $a \cup b$ and $a \cap b$ may also be considered to be the result of two algebraic operations with certain simple properties. Recent investigations have shown that many mathematical theories may be formulated in terms of such structures, and the systematic use of these concepts gives a unification and a simplification of the various theories. As special instances one may mention the set theory of Stone (Harvard), projective geometry by Garrett Birkhoff (Harvard) and von Neumann (Institute for Advanced Study), euclidean and non-euclidean geometry by Menger (Notre Dame), algebraic theories by the lecturer. Among the many other recent contributions to lattice theory, one can mention papers by Dilworth (Yale) and Ward (California Institute of Technology) on multiplication in such systems, by MacLane (Harvard) on certain types of lattices applicable to chains in algebraic systems, by Glivenko on metric lattices, by Jacotin-Dubreils on equivalence relations. In these lectures only certain specific problems, mainly of an algebraic nature, can be discussed with some completeness.

GARRETT BIRKHOFF

Saunders MacLane

OYSTEIN ORE

\section{CONFERENCE ON THE THEORY OF INTEGRATION}

The sessions of this conference were held concurrently with those of the Conference on Algebra. The following paragraphs give a relatively inadequate summary of the lectures, which provided a very illuminating introduction to the researches of the various speakers, especially in their relation to the historical development of the theory of integration.

The first session was devoted to theories of integration in abstract spaces, and both speakers were concerned with extreme generalizations of the notion of the upper and lower integrals of Darboux. Professor Bochner, in his lecture entitled Integration and partial ordering, 
discussed the problem of extending the domain of a positive and finitely additive operator $M$ on $V$ to $Y$, when $V, V^{\prime}$ and $Y$ are partially ordered vector spaces with $V \subset V^{\prime}$, and $Y$ is also assumed to be a complete lattice. For an arbitrary element $\phi$ of $V^{\prime}$ the extension $M \phi$ satisfies the inequalities

$$
M_{*} \phi \equiv \sup _{f \leqq \phi} M f \leqq M \phi \leqq \inf _{f \geqq \phi} M f \equiv M^{*} \phi,
$$

where $f$ ranges over $V$. The largest subset of $V^{\prime}$ on which the extension of $M$ is uniquely determined is the set $\bar{V}$ on which $M^{*} \phi=M_{*} \phi$. The space $\bar{V}$ is complete in the Riemann-Darboux sense, that is, if $\theta$ in $V^{\prime}$ and $\phi_{n}$ and $\psi_{n}$ in $\bar{V}$ are such that

$$
\phi_{n} \leqq \phi_{n+1} \leqq \theta \leqq \psi_{n+1} \leqq \psi_{n}, \quad \sup M \phi_{n}=\inf M \psi_{n},
$$

then $\theta$ belongs to $\bar{V}$. In certain special cases, when the elements of $V^{\prime}$ are point functions, and the space $\bar{V}$ contains sufficiently many step-functions for purposes of approximating to $M \phi$ for arbitrary $\phi$ in $\bar{V}$, the operation $M \phi$ may appropriately be considered as an integral $\int \phi(x) d \mu(x)$. Bochner showed how the theory may be applied to a generalization of the classical moment problem, that is, to secure a representation of a completely monotone function $f(x)$ with values in $Y$ in the form

$$
f(x)=\int_{0}^{\infty} e^{-x t} d \mu(t) .
$$

Among other applications mentioned was the spectral resolution of a bounded hermitian operator in Hilbert space. The discussion of Bochner's paper was initiated by Dr. R. S. Phillips.

Professor Price, in his lecture entitled Integration and convex operators, began with an historical discussion of the use of the upper and lower sums of Darboux, leading up to the generalization of this notion in the "integral range" of Garrett Birkhoff. Later developments in this direction by Price himself required the use of a generalized convex operator. In the present discussion the speaker showed that the notion of a convex operator is entirely unnecessary. In contrast to the approach of Bochner outlined above, Price assumes a "measure function" $T_{\sigma}$ given in advance, in terms of which an integral operator $M(f)=\int f d T$ is constructed. No partial ordering of the domain or the range of $M$ is used. The integral range is constructed as follows. Let $V$ be a vector space, $S$ a class of elements $\sigma$, and $f$ a function of $\sigma$ whose values are subsets of $V$. Let $T_{\sigma}$, for each $\sigma$ in $S$, be an additive transformation of $V$ into $V$, let $\Delta$ denote a finite subset $\left(\sigma_{1}, \cdots, \sigma_{n}\right)$ of $S$, and let $D$ be a class of such subsets $\Delta$ which constitutes a directed 
set in the sense that it is partially ordered and has the composition property. The integral range of $f$ associated with $\Delta$ is defined by the formula

$$
I(f, \Delta)=\sum_{\Delta^{\prime} \geqq \Delta}\left[T_{\sigma_{1}^{\prime}} f\left(\sigma_{1}^{\prime}\right)+\cdots+T_{\sigma_{n}^{\prime}} f\left(\sigma_{n}^{\prime}\right)\right],
$$

where $\Delta^{\prime}=\left(\sigma_{1}^{\prime}, \cdots, \sigma_{n}^{\prime}\right), \sum$ denotes the logical sum, and + denotes the addition of complexes. This integral range obviously has the following properties: (1) $\Delta_{2}>\Delta_{1}$ implies $I\left(f, \Delta_{2}\right) \subseteq I\left(f, \Delta_{1}\right)$; (2) $I\left(f_{1}+f_{2}\right.$, $\Delta) \subseteq I\left(f_{1}, \Delta\right)+I\left(f_{2}, \Delta\right)$. No topological properties of the space $V$ enter in to the definition of $I(f, \Delta)$. However, the method of defining a class of integrable functions and an integral $\int f d T$ associated with the class of integral ranges $[I(f, \Delta)]$ depends on the topology in $V$. If $V$ is a Banach space, we may say that $f$ is integrable in case the lower bound of the diameters of its integral ranges is zero, and $\int f d T$ is then the unique element common to the closures of the integral ranges of $f$. The integral has the usual properties when suitable restrictions on the basis are made. The speaker indicated the solution of this topological problem of integration also in the case when the space $V$ is a space $(L)$ of Fréchet. Professor Nelson Dunford initiated the discussion of this paper.

Professor Hildebrandt began his lecture on The general integral of E. H. Moore by pointing out the three-fold character of Moore's investigations into theories of integrals. Moore first interested himself in improper integrals of the Harnack type and then in his two forms of general analysis. The central feature of both forms is the introduction of a completely unrestricted range $\mathfrak{B}$ on which is defined the class $\mathfrak{M}$ of complex- (or real-) valued functions to be discussed. The first form of general analysis, a group of postulational theories, was an attempt to correlate certain theories of linear equations, including the Fredholm theory. In one formulation of this analysis two classes $\mathfrak{M}_{1}$ and $\mathfrak{M}_{2}$ of the type mentioned above are considered, and the integral is a bilinear, continuous operator, continuity being defined with respect to a relative uniform convergence topology. In the case $\mathfrak{M}_{1}=\mathfrak{M}_{2}$, the operator $J$ may be taken to be properly positive and hermitian and is thus the forerunner of the basis of a general Hilbert space.

To illustrate how the second form was developed Hildebrandt discussed the Schmidt theory of the system of equations

$$
\sum_{i=1}^{\infty} x_{i} a_{i j}=y_{j}, \quad i=1,2, \cdots,
$$


and then indicated the extension to the case when the variables $i$ and $j$ have general ranges. For functions $\mu_{1}, \mu_{2}$ of a class of "modular functions" an integral $J_{\bar{\mu}_{1} \mu_{2}}$ is defined which is a bilinear, properly positive and hermitian operator. If $J \bar{\mu}_{1} \mu_{2}$ is taken to be the inner product $\left(\bar{\mu}_{1}, \mu_{2}\right)$, the space of modular functions is seen to be a generalized Hilbert space (with no restriction on the dimension number). Among the important features of Moore's general analysis from the point of view of present day mathematics are his use of a completely unrestricted domain $\mathfrak{B}$ for his modular functions, his conception of an integral as a bilinear operator, and his exploitation of the MooreSmith limit, which has led to the modern use of directed sets. In discussing the paper Professor Barnard, among other things, developed the modular space determined by functions $f(x-y)$ of a type discussed in the previous lecture by Bochner.

Professor Jeffery began his discussion of Non-absolutely convergent integrals with a brief historical summary of the early work of Denjoy and Perron, recalling that it was not until 1924 that Alexandroff and Looman showed the equivalence of the Perron integral with the special Denjoy integral. The problem of formulating an integral of Perron type equivalent to the general Denjoy integral was solved by Ridder in 1933. The speaker gave Ridder's definition and an outline of the equivalence proof. He next discussed the approximately continuous integral of Burkill and also his "Cesàro-Perron" integral of the first order, of all positive integral orders, and finally of all real orders, with a brief description of the application of Burkill's work to Fourier series. For a list of Burkill's papers on these subjects the reader is referred to volume 11 of the Journal of the London Mathematical Society.

In conclusion Jeffery discussed an approach to the problem of nonabsolutely convergent integrals which was originated by himself and M. S. Macphail. A function $F(e)$ of the measurable sets $e$ on the interval $(a, b)$ is absolutely-additive relative to the closed set $E$ if it is completely-additive over $E$ and if for the intervals $\left(\alpha_{i}, \beta_{i}\right)$ complementary to $E, \sum F\left(\alpha_{i}, \beta_{i}\right)=F\left[\sum\left(\alpha_{i}, \beta_{i}\right)\right] . F(e)$ is generalized absolutelyadditive on $(a, b)$ if this interval can be covered by a denumerable sequence of closed sets relative to each of which $F(e)$ is absolutelyadditive. Let $f(x)$ be measurable and let $s_{n}(x)$ be a sequence of summable functions tending to $f(x)$ such that $\int_{a}^{x} s_{n} d x$ tends to a limiting function $F(x)$. There is thus associated with $F(x)$ a function $F(e)$ $=\lim \int_{e} s_{n} d x$, provided this limit exists. If it turns out that $F(e)$ is generalized absolutely-additive on $(a, b)$, then $F(x)$ is the non-absolutely convergent integral of $f(x)$. This integral is equivalent to the 
general Denjoy integral. The approach lends itself to a simple and brief treatment which quickly brings out all the main facts about nonabsolutely convergent integrals. It is also easily adapted to integrals of the Stieltjes type, and to a formulation of a theory of non-absolutely convergent integrals in abstract space. The discussion of Jeffery's lecture was led by Professor McShane.

In introducing his talk on Measure in statistical mechanics Professor Wiener considered a measure-conserving mapping $T$ of the interval $(0,1)$ into itself and remarked that the set $\Sigma$ of points $P$ for which $a_{i} \leqq T^{i} P<b_{i}(i=0, \pm 1, \pm 2, \cdots, \pm n)$ is measurable. As a consequence, then, of the ergodic theorem, it follows that $\lim _{N=\infty}$ (Number of values of $k$ in $(1 ; N)$ for which $T^{k} P$ is in $\Sigma$ ) $/ N=m \Sigma$ and for almost all values of $P$ this will be true for all such sets $\Sigma$ simultaneously. A sequence $\left\{x_{k}\right\}(k=0, \pm 1, \pm 2, \cdots)$ is called by Wiener a time series, and it is termed regular if the number of values of $k$ in $(-N,+N)$ for which $a_{i} \leqq x_{k-i}<b_{i}(i=0, \pm 1, \pm 2, \cdots, \pm n)$, when divided by $2 N$, tends to a limit for every selection of the $a_{i}, b_{i}$. It was indicated above how the time series $T^{i} P(i=0, \pm 1, \cdots)$ is regular. Next Wiener showed how every regular time series generates a measure of time series together with a transformation under which this measure is invariant, or in other words establishes a situation under which ergodic theory may be applied. It may be shown that almost all time series are regular and generate the same measure among time series. Thus with respect to this measure, translation in time is metrically transitive. It can be shown that any measure-preserving transformation may be broken up into metrically transitive components. One of the greatest needs of ergodic theory is the technique of producing measure-preserving transformations with desired spectrum characteristics. It is generally desired that these transformations be mutually transitive. One of the easiest ways of producing such transformations is the explicit formation of regular time series. Wiener's theory of Brownian motion was in essence nothing but a proof that almost all Brownian motions generate the continuous analogue of a regular time series. Another interesting application of time series is in the theory of almost periodic functions. Wiener next gave a particularly interesting example due to himself and Kurt Mahler to show the existence of time series with non-absolutely continuous spectra. It is known that the metrically transitive transformation generated by this example is not even mixing in the weak sense, that is, the distribution of $x_{k}, \cdots, x_{k+j}$ is not even on the average independent of that of $x_{k+l}, \cdots, x_{k+l+j}$, where $l>j$. On the other hand, let $\left\{p_{i}\right\}$ be an increasing sequence of primes. Represent a number $N$ in the form 
$A_{0}+\sum_{1}^{N} A_{k} p_{1} \cdots p_{k}\left(A_{k}<p_{k+1}\right)$ and let $x_{ \pm n}=\prod_{0}^{N} \omega_{k} A_{k}(n>0)$, where $\omega_{k}$ is a primitive $p_{k+1}$ th root of unity. Then the time series $x_{n}$ will be regular and the translation operator will be metrically transitive with respect to the measure thus generated. It will in addition be weakly mixing but is not strongly mixing. Wiener concluded his address by conjecturing that a slight generalization of such methods may be used to derive strongly mixing transformations which will be such that the spectrum (in the sense of his generalized harmonic analysis) of some sequence $f\left(T^{N} P\right)$ will be non-absolutely continuous. This would indicate that not all mixing transformations are isomorphic. The discussion was initiated by Professor von Neumann.

The talk on Product integrals by Professor Garrett Birkhoff began with a brief discussion of the solution, in terms of a product integral, of a system of ordinary linear differential equations, as developed by Volterra and Schlesinger. Here the matrix product replaces the ordinary sum in the definition of the integral. Birkhoff has generalized this notion, defining a product integral for functions whose values are in a complete normed vector ring with a unit. Thus, let $V(t)$ be defined on the interval $(a, b)$, and let $\Pi$ be a partition $\left(\Delta_{1}, \Delta_{2}, \cdots\right.$, $\Delta_{n}$ ) of this interval. Then the Riemann product integral is defined by the equation

$$
\int_{a}^{\widehat{b}} V(t) d t=\lim _{\pi}\left[V\left(t_{1}\right) \Delta_{1} \bigcirc V\left(t_{2}\right) \Delta_{2} \bigcirc \cdots \circ V\left(t_{n}\right) \Delta_{n}\right],
$$

where $t_{k}$ is in $\Delta_{k}$, provided the limit exists in the Moore-Smith sense. The ring operation $\bigcirc$ must be distinguished from the "scalar multiplication" by $\Delta_{k}$. It should be noted that when the operation $O$ is interpreted as ordinary addition, the process of product integration just defined reduces to integration in the ordinary sense. Birkhoff indicated that while the notion of product integral may clearly be applied to linear operators on any Banach space, it may also be so interpreted as to apply to non-linear operators. In this case two norms are needed in the space of operators. The distributive law which holds in any ring may be replaced by approximate distributivity (that is, differentiability) near the unity element. In the finite-dimensional case, this permits the integrands to have values in any Lie group; the matrix-valued integrands of Volterra and Schlesinger are thus included as a very special case. In the infinite-dimensional case, the values can be in any "analytical group" (see Birkhoff's paper in Journal of Mathematics and Physics, vol. 16 (1937), pp. 104-132). An extension of the notion of product integral indicated above to one of 
Lebesgue type was discussed by the speaker. Certain properties have been secured, but many other problems remain to be elucidated. The connection between product integrals and the fundamental group in the simplest case of a surface was exhibited by Professor Rainich, whose remarks were communicated in a letter read to the conference.

The lecture on Applications of the theory of integration to the study of linear operators by Professor Dunford stated some of the unsolved problems as well as the known facts about one type of representation of linear maps between Lebesgue spaces, and also gave an outline of the method of Dunford and Pettis for attacking these problems. If $\psi=U \phi$ is a bounded linear map of $L^{p}(T)$ onto $L^{q}(S)$, where $S$ and $T$ are finite intervals of the real axis, it has a representation of the form

$$
\psi(s)=\frac{d}{d s} \int_{T} H(s, t) \phi(t) d t .
$$

In case $p=1$ and the map $U$ is weakly compact (that is, $U$ takes the unit sphere into a set whose closure is weakly compact), it has the simpler representation

$$
\text { - } \psi(s)=\int_{T} K(s, t) \phi(t) d t .
$$

However, in case $p=q=1$ there are maps of the form (2) which are not weakly compact, and a topological characterization of the integral operator (2) between $L$ spaces is unknown. One of the results stated by Dunford in this connection asserts that (2) (with $p=1 \leqq q$ ) takes weakly compact sets into compact sets. This shows that the product of two weakly compact operators in $L$ is a compact operator and makes it clear why certain aspects of the Fredholm theory hold for linear equations involving a weakly compact operator in $L$.

A fundamental class of problems concerning the operators (1) and (2) involves the characterizing in terms of the kernels $H$ and $K$ of those operators $U$ which are bounded, weakly compact, compact, lattice convergence-preserving, and so on. These problems have for the most part been solved in case $p=1 \leqq q$ even in the case of abstract $S$ and $T$, but in all other cases very little is known. One method of investigation which has proved fruitful in the case $p=1$ consists of the following threesteps : first, represent the operator $U$ as an abstract integral $U \phi=\int_{T} \phi(t) x(t) d t$; second, find the relations between the set $J=\left[(1 /|e|) \int_{e} x(t) d t\right]$ and the set of values $x(T)$ of the kernel; third, express the abstract integral as a numerical integral, that is, $\int_{e} x(t) d t$ $=\int_{e} K(s, t) d t$ where $x(t)=K(\cdot, t)$. For bounded operators $U$ on $L$ to 
$X$ the first problem is solvable with a Gelfand integral in case $X$ is the adjoint of a separable space and with a Bochner integral (for arbitrary $X$ ) in case $U$ is weakly compact. The principal result under the second problem is that for measurable Pettis integrable functions there is a null set $E_{0} \subset T$ such that $x\left(T-E_{0}\right)$ and $J$ have the same closed convex hull. The representation desired in the third step always holds for Bochner integrable functions and at least for those Gelfand integrable functions arising in the representation of linear operations on $L(T)$ to $L^{q}(S)$. Clearly, it is the second step above which allows us to state the topological properties of the operator (at least those which are invariant under the operation of convex closure) in terms of the kernel. A partial list of those papers treating the problems mentioned above is contained in a paper by Dunford and Pettis appearing in the Transactions of this Society, vol. 47 (1940), pp. 323-392. Dunford's paper was discussed by Professor J. W. Calkin.

H. H. Goldstine

L. M. Graves 